\title{
Contextualized Personality Questionnaires: A Case for Copulas in Structural Equation Models for Categorical Data
}

\author{
Johan Braeken \\ Research Methodology Group, Wageningen University and Research \\ Centre, The Netherlands \\ Quantitative Psychology and Individual Differences, \\ $K U$ Leuven-University of Leuven, Belgium \\ Peter Kuppens \\ Quantitative Psychology and Individual Differences, \\ KU Leuven-University of Leuven, Belgium \\ Paul De Boeck \\ Quantitative Psychology and Individual Differences, \\ $K U$ Leuven-University of Leuven, Belgium \\ Department of Psychology, Ohio State University \\ Francis Tuerlinckx \\ Quantitative Psychology and Individual Differences, \\ KU Leuven-University of Leuven, Belgium
}

\begin{abstract}
For structural equation models (SEMs) with categorical data, correlated measurement residuals are not easily implemented. The problem lies mainly in the absence of a categorical analogue to the multivariate normal distribution and the absence of closed form formulas in SEMs for categorical data. We present a novel technique to handle measurement residuals that keeps the attractive SEM mainframe intact yet
\end{abstract}

Correspondence concerning this article should be addressed to Johan Braeken, Wageningen University and Research Centre, Education and Competence Studies, Research Methodology Group (Bode 68), P.O. Box 8130 - 6700 EW, Wageningen, The Netherlands. E-mail: j.braeken@ flavus.org 
adds flexibility in dependence modeling without excessive computational burden. The technique is based upon the concept of copula functions and is introduced with a data set of ordinal responses originating from a contextualized personality study on aggression. Focus is on models arising in a multitrait-multimethod context, where the flexibility in dependence structures allows for method effects that can vary across the latent trait dimension. The empirical application illustrates that ignoring design-implied correlated measurement residuals can potentially influence study results and conclusions in both a quantitative as well as a qualitative way. Model parameter estimates can be biased, but more important, model inferences can be heavily distorted.

Traits are an important conceptual device in personality psychology and the study of individual differences. A trait is defined as a relatively permanent individual characteristic that is generalizable across situations and contexts, and forms the foundation of a person's typical behavior. Yet, since Mischel (1968; also see Mischel \& Shoda, 1995) demonstrated the lack of behavioral situational consistency, a long-standing debate ensued about the importance of such traits because behavior occurs in a context and is not determined only by intrinsic personal characteristics. An important lesson drawn from this debate is that it is important to acknowledge the context in which behavior takes place. This change in the understanding and position of traits has also influenced the measurement practice. Taking into account the context of behavior, it makes sense to assess and study personality not in a vacuum (i.e., with contextless general indicators) but to use a measurement instrument that looks at different situations that provide relevant context for a person's behavior on a particular trait, such as in contextualized personality questionnaires (see, e.g., Vansteelandt \& Van Mechelen, 1998).

Research that seeks to validate a measurement instrument or test theories about relations among traits often makes use of confirmatory factor analysis (CFA) or the more general structural equation models (SEMs). In such a model, we assume that indicator variables are manifestations of a smaller set of latent trait factors, explaining the common information shared by items of the same trait, and a residual part that can be considered to contain a combination of measurement error and item-specific factors. The key assumption here is that the latent traits are considered sufficient to explain the association between the individual outcomes. This is often referred to as the conditional independence assumption. However, in contextualized questionnaires, several indicators may refer to the same situation and this creates additional residual association beyond what is accounted for by latent personality traits. Cole, Ciesla, and Steiger (2007) convincingly showed that problems arise when not all residual association that is implied by the measurement strategy or research design is included in the model. Often these situations come down to a research design that is similar to a (complete or incomplete) multitrait-multimethod design. Two main 
sources of dependence appear, item indicators measuring the same trait will show dependence due to population heterogeneity on this dimension of interest, yet at the same time a counfounding additional source of dependence is at work, attributed to another shared research design element (e.g., similar datagathering method, similar information source, same rater). One might carry out exploratory analyses to investigate whether residual dependencies exist by examining bivariate or higher order measures of association between residuals or try a specification search based upon modification indices (for a critical note see, e.g., MacCallum, 1986; Salhi, 1998; Steiger, 1990). However, when there are theoretical reasons for the possibility of residual dependencies and they are of interest, as in the contextualized personality case, a confirmatory model comparison approach is a more sound and direct alternative.

In the applied SEM literature, two approaches to deal with residual association are commonly used: the correlated trait factors-correlated method factors (CT-CM) model and the correlated trait factors-correlated uniqueness (CT-CU; see, e.g., D. Kenny, 1976; H. Marsh, 1989). The CT-CM approach takes the perspective that what underlies the additional residual associations are other individual difference factors-so-called method factors-that are uncorrelated to the trait factors. Although the logic of the approach is straightforward, there is a practical downside in adding more and more latent factors to the model. Especially in cases with a limited number of indicators, the CT-CM approach can easily end up in an overfactorization of a given data set, resulting in exact and/or empirical model identification problems (see, e.g., Eid, 2000; Kenny \& Kashy, 1992; Rindskopf, 1984; Wothke, 1996). The other common approach, the CT-CU model, accommodates the residual association issue by relaxing the requirement that measurement residuals of indicators must be independent and allowing specific pairs of measurement residuals to correlate within the model. The CT-CU approach is more ad hoc but does have practical advantages over the addition of extra method factors (i.e., the CT-CM approach) in a sense that it is better identified, more stable, and less likely to run into computational problems. The downside is that the CT-CU model is misspecified when the methods are nonorthogonal and that it is less parsimonious for studies with many indicators. Note that a more extensive discussion (and other variations) of these models can be found in the work of Lance, Noble, and Scullen (2002) and Eid et al. (2008). Both CT-CM and CT-CU share the common limitation that no interplay between methods and traits is allowed. The dependence due to the methods and dependence due to the traits are fully orthogonal. In the study of personality, this might not be realistic. Introverted people, for instance, will appear to be even more introverted when observed in a public situational context than in a home context, whereas the specific situational context might not affect more extraverted people. Hence, method effects might vary across the latent trait dimension. 
SEMs were developed as extensions of classical linear regression and path analysis and, hence, they are more or less tailor-made for the case of normally distributed variables. To accommodate categorical variables, a similar logic can be followed as in extending the linear regression model to the generalized linear model (see, e.g., Skrondal \& Rabe-Hesketh, 2004). However, whereas in the case of normal distributed variables the variance-covariance matrix and mean structure are sufficient statistics to describe their distribution (Knol \& Berger, 1991; Mood, Graybill, \& Boes, 1974), there is no such direct analogue in the case of categorical variables. This makes the computational algorithms in SEM for categorical data much more complex (for a recent overview see, e.g., Wirth \& Edwards, 2007). Thus from a computational perspective, the problems with the CT-CM approach are even more persistent in the categorical data case than in the continuous data case. The computational challenge is not limited to the CT$\mathrm{CM}$ approach but also applies to the CT-CU approach. The problem lies mainly in the absence of a categorical analogue to the multivariate normal distribution and the absence of closed form formulas in SEMs for categorical data. Hence, in the context of categorical data, the implementation of correlated measurement residuals is not straightforward.

Our goal is to present an approach similar to the CT-CU approach but with added flexibility in specifying different types of residual dependence that do not necessarily require the residual dependence to be completely orthogonal to the latent trait. Thus, the dependence between measurement residuals for items that refer to the same context might be stronger for someone with a more pronounced personality trait. Given the small sample sizes and complex models often used in behavioral sciences, a parsimonious structure is be pursued that allows for straightforward interpretations without excessive computational burden. Our approach is based on copula functions as they have been introduced recently in the psychometric literature by Braeken, Tuerlinckx, and De Boeck (2007) and by Braeken and Tuerlinckx (2009) in the context of item response theory and mixed models. In this article, we extend their method to handle ordered polytomous data.

\section{ORGANIZATION OF THE ARTICLE}

We start with a brief introduction of copula functions as multivariate link functions that build multivariate distributions from given margins. A copula variant of a CT-CU model for categorical manifest indicators is defined and shown to have new attractive possibilities in an application involving a contextualized personality study that investigates the ABC model for aggression. The application illustrates that ignoring correlated measurement residuals that can be expected to arise due to the study design can potentially influence the study results and conclusions in both a quantitative and a qualitative way. 


\section{COPULA FUNCTIONS}

The history of copula functions goes back to the 1940s in the context of probabilistic metric spaces. Copula functions were introduced to study dependence measures and margin-free properties of dependence. We limit our exposition to properties that are essential for their use in statistical modeling (for an extensive overview of copula theory, see Nelsen, 1999, or Joe, 1997). Fundamentally, copula functions are a set of multivariate distributions with univariate margins that are defined on the [0,1] interval: $C(\boldsymbol{U})=\left(U_{1}, \ldots, U_{i}, \ldots, U_{I}\right)$ with each $U_{i} \sim \operatorname{Unif}(0,1)$. The use of copula functions in statistical modeling is made clear by Sklar's theorem (Sklar, 1959), which defines a copula as a kind of multivariate link function (cf. link functions in the generalized linear model). The theorem builds on the familiar inversion method and is based on the fact that any univariate cumulative distribution function (CDF) $F_{Y_{i}}$ for an outcome variable $Y_{i}$ is defined on the $[0,1]$ interval. By inversion of the following transformation $U_{i}=F_{Y_{i}}$ the theorem establishes two important results. First, the representation result of the theorem states that any multivariate $\mathrm{CDF} F_{\boldsymbol{Y}}$ with univariate margins $F_{Y_{1}}, \ldots, F_{Y_{I}}$ can be reformulated as a function of these margins by means of a copula function $C$. In the continuous case, this reformulation is unique; in the discrete case, it is uniquely determined on the range of the margins.

$$
F_{\boldsymbol{Y}}=C\left(F_{Y_{1}}, \ldots, F_{Y_{I}}\right) .
$$

This side of the theorem is especially useful for the mathematical study of dependence properties. Second, the construction result of the theorem states that a given set of univariate margins $F_{Y_{1}}, \ldots, F_{Y_{I}}$ can be coupled together by means of a copula function $C$ to construct a new multivariate distribution with given margins. The following scheme illustrates the basic principle of "change in variables":

$$
\begin{gathered}
\left(U_{1}, \ldots, U_{i}, \ldots, U_{I}\right) \sim C(\boldsymbol{U}) \\
\underbrace{\left(Y_{1}, \ldots, Y_{i}, \ldots, Y_{I}\right) \sim F_{\boldsymbol{Y}} .}_{\begin{array}{c}
\uparrow \\
U_{i}=F_{Y_{i}}\left(y_{i}\right) \\
Y_{i}=F_{Y_{i}}^{-1}\left(U_{i}\right)
\end{array}}
\end{gathered}
$$

The resulting multivariate probability density function built under the copula approach factorizes as a product of univariate densities and the copula density

$$
f(\boldsymbol{Y})=c\left(F_{Y_{1}}, \ldots, F_{Y_{I}}\right) \prod_{i=1}^{I} f\left(Y_{i}\right) .
$$


Hence, Sklar's theorem opens up the possibility of more tailor-made models. Because the copula construction preserves the univariate margins (i.e., $\left.C\left(1, F_{Y_{i}}, 1\right)=F_{Y_{i}}\right)$, one can start the modeling approach by searching for univariate margins that are well suited for the problem at hand and then proceed by constructing the joint dependence model by coupling these margins with a copula function. Although a whole set of univariate distributions exist and are readily available, most have no natural extension to the multivariate case. Thus, most researchers would be forced to use the default multivariate normal distribution. Copulas allow you to expand your view and, for instance, build a bivariate model for a variable $Y_{1}$ that is beta distributed and a variable $Y_{2}$ that follows a lognormal distribution. Furthermore, adopting a different copula function to couple the two variables doesn't change their univariate distribution but does change the implied dependence structure. Figure 1 illustrates this by showing four scatterplots each of two standard normal variables $X$ and $Y$ but connected by different copula functions. In the bottom-right panel, the familiar bivariate normal distribution is recognized. This is in fact an instance of the socalled Gaussian copula $\left(C_{\Phi}\right)$ with two standard normal margins. The upper left panel also shows a symmetric dependence pattern, which is more squared than elliptical. This is an instance of the Frank copula $\left(C_{F}\right.$; Frank, 1979). The upper right and bottom-left panel show dependence patterns in which the dependence is stronger in the right tail or left tail of the joint distribution. These are instances of the Gumbel-Hougaard $\left(C_{G H}\right.$; Gumbel, 1960; Hougaard, 1986) and CookJohnson copula $\left(C_{C J}\right.$; Clayton, 1978; Cook \& Johnson, 1981), respectively. Thus, we have three bivariate distributions that are not bivariate normal but do have normal margins. Mathematical formulas for the copulas being used can be found in the Appendix.

In the next section, we define the SEM framework for categorical data and show how to incorporate copula functions to account for dependence among measurement residuals. The basic idea is that we couple the conditional response probability functions of two residually dependent indicators to construct a joint conditional distribution that allows for dependence.

\section{THE COPULA CT-CU VARIANT FOR CATEGORICAL DATA}

If participant $p(p=1, \ldots, P$ with $P=340)$ judges the plausibility of a specific reaction on an indicator $i(i=1, \ldots, I$ with $I$ and $I=9)$ in category $q$ (with values $q=0, \ldots, Q-1$ and $Q=3$ ), then we write $Y_{p i}=q$. Because of the ordinal response scale, the plausibility of the reaction increases with increasing category level $q$. The modeling proceeds with assuming that the 


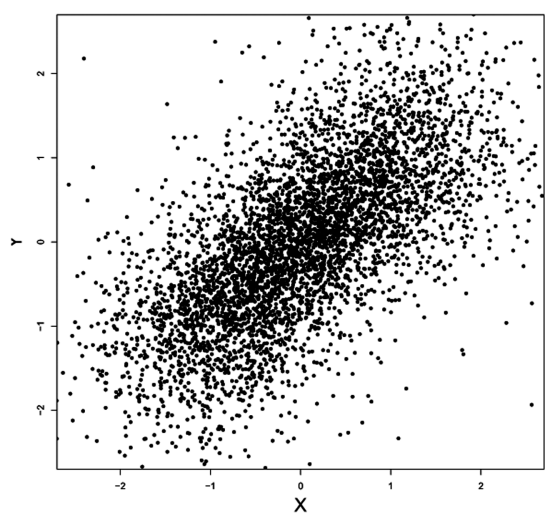

(a)

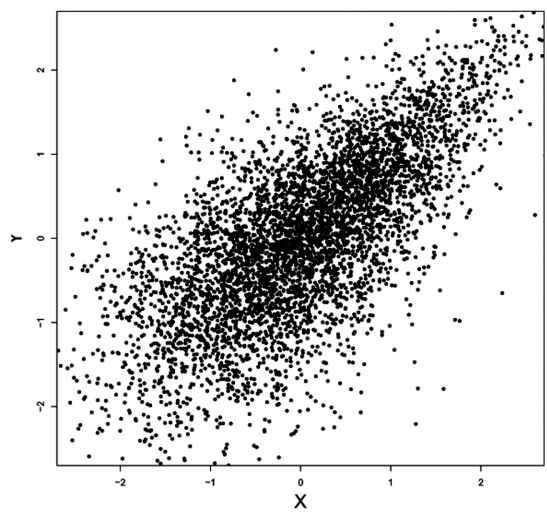

(c)

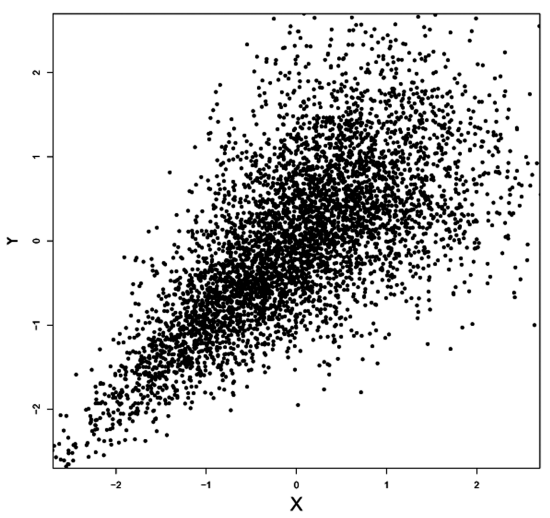

(b)

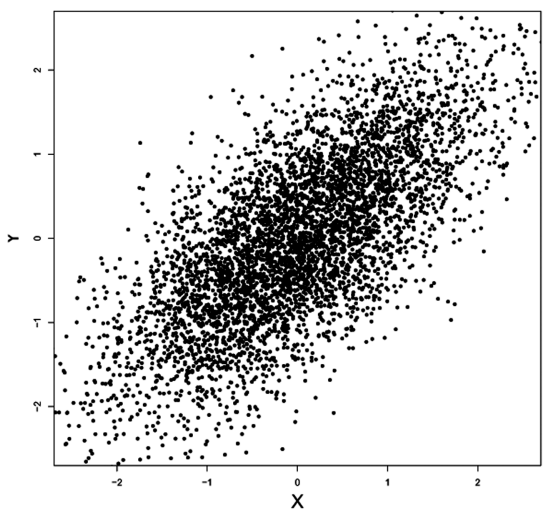

(d)

FIGURE 1 Copula quartet of bivariate distributions with standard normal margins. (a) Frank copula. (b) Cook-Johnson copula. (c) Gumbel-Hougaard copula. (d) Gaussian copula.

categorical ordinal outcome $Y_{p i}$ is a discrete manifestation of an underlying latent continuous variable $\tilde{Y}_{p i}$ according to a threshold model

$$
Y_{p i}=q \quad \text { if } \quad \tau_{i q}<\tilde{Y}_{p i} \leq \tau_{i, q+1}
$$

where the thresholds satisfy the ordinal restriction:

$$
-\infty=\tau_{i 0} \leq \tau_{i 1} \leq \ldots \leq \tau_{i q} \leq \ldots \leq \tau_{i, Q-1} \leq \tau_{i Q}=+\infty
$$




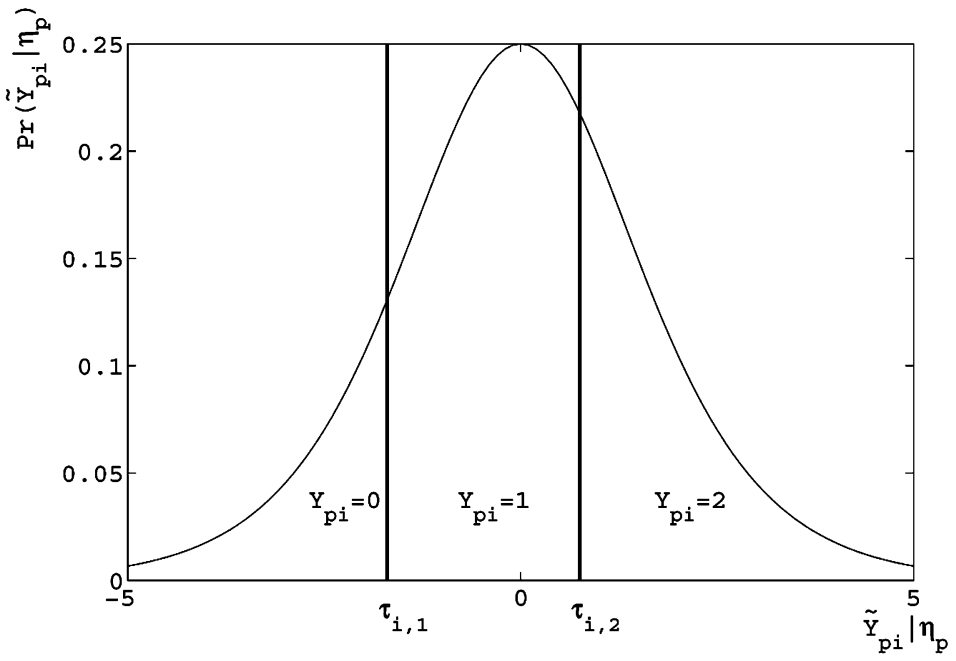

FIGURE 2 Threshold model for the latent outcome $\tilde{Y}_{p i}$.

This idea is depicted graphically in Figure 2 for $Q=3$. This formulation in terms of continuous underlying latent variables $\tilde{\boldsymbol{Y}}_{p}$ enables the application of traditional measurement and structural models on this latent level.

\section{MEASUREMENT MODEL}

Consider the following measurement model for the vector of latent outcomes $\tilde{\boldsymbol{Y}}_{p}$ :

$$
\begin{aligned}
\tilde{\boldsymbol{Y}}_{p} & =\boldsymbol{\Lambda} \boldsymbol{\eta}_{p}+\boldsymbol{\varepsilon}_{p} \\
\boldsymbol{\eta}_{p} & \sim N(\mathbf{0}, \boldsymbol{\Sigma}) \\
\boldsymbol{\varepsilon}_{p} & \sim \operatorname{Logist}(\mathbf{0}, \boldsymbol{\Psi}) .
\end{aligned}
$$

The $D \times 1$ matrix $\eta_{p}$ is the common factor score matrix, with $\eta_{p d}$ the score of person $p$ on common factor $d(d=1, \ldots, D)$. The common factors are assumed to follow a multivariate normal distribution with covariance matrix $\boldsymbol{\Sigma}$. The $I \times D$ matrix $\Lambda$ is the factor loading matrix, with $\lambda_{i d}$ the factor loading of indicator $i$ on common factor $d$. The $I \times 1$ matrix $\varepsilon_{p}$ is the measurement residual matrix, with $\varepsilon_{p i}$ the unique factor score or measurement residual for indicator $i$ of person $p$. The measurement residuals follow a logistic distribution with a diagonal covariance matrix $\Psi$. Hence, the $I$ residuals are independent and this is 
the conditional independence assumption of the model: the set of latent common factors is assumed to completely explain the association between the outcomes. The usual set of restrictions applies to identify the model and comes down to setting the metric of the latent variables in a standardized format (Bartholomew \& Knott, 1999).

This setup gives rise to the following conditional probability formulation for an individual outcome given a latent factor score vector $\boldsymbol{\eta}_{p}$ :

$$
\begin{aligned}
\operatorname{Pr}\left(Y_{p i} \geq q \mid \boldsymbol{\eta}_{\boldsymbol{p}}\right) & =\operatorname{Pr}\left(\tilde{Y}_{p i} \geq \tau_{i q} \mid \boldsymbol{\eta}_{p}\right) \\
& =1-\operatorname{Pr}\left(\tilde{Y}_{p i}<\tau_{i q} \mid \boldsymbol{\eta}_{p}\right) \\
& =1-\operatorname{Pr}\left(\varepsilon_{p i}<\tau_{i q}-\sum_{d=1}^{D} \lambda_{i d} \eta_{p d}\right. \\
& =1-\operatorname{Pr}\left(\varepsilon_{p i}^{*}<\left(\tau_{i q}-\sum_{d=1}^{D} \lambda_{i d} \eta_{p d}\right) / \sqrt{\Psi_{i}}\right) \\
& =G\left[-\left(\tau_{i q}-\sum_{d=1}^{D} \lambda_{i d} \eta_{p d}\right) / \sqrt{\psi_{i}}\right],
\end{aligned}
$$

where $\epsilon_{p i}^{*}$ follows a logistic distribution with variance 1 and its CDF is denoted by $G$ (such that $G(x)=\frac{e^{\frac{x}{k}}}{1+e^{\frac{1}{k}}}$, where $k=\frac{\sqrt{3}}{\pi}$ ).

The factor analysis model can be reparametrized such that one obtains a formulation akin to item response models (see, e.g., Takane \& de Leeuw, 1987). This reparametrization is carried out by absorbing the variance of the measurement residual $\varepsilon_{p i}$ (the unique variance $\psi_{i}$ ) into the thresholds and loadings:

$$
\begin{aligned}
\operatorname{Pr}\left(Y_{p i} \geq q \mid \eta_{p}\right) & =G\left[-\left(\tau_{i q}-\sum_{d=1}^{D} \lambda_{i d} \eta_{p d}\right) / \sqrt{\psi_{i}}\right] \\
& =\frac{\exp \left(\sum_{d=1}^{D} \alpha_{i d} \eta_{p d}-\beta_{i q}\right)}{1+\exp \left(\sum_{d=1}^{D} \alpha_{i d} \eta_{p d}-\beta_{i q}\right)},
\end{aligned}
$$

where the new parameters are

$$
\alpha_{i d}=\frac{\tau_{i q}}{\sqrt{1-\lambda_{i}^{\prime} \Sigma \lambda_{i}}} \quad \text { and } \quad \beta_{i q}=\frac{\tau_{i q}}{\sqrt{1-\lambda_{i}^{\prime} \Sigma \lambda_{i}}} .
$$

Both parametrizations are equivalent, yet for estimation purposes, we work with the unstandardized coefficients $\alpha$ and $\beta$ in the conditional probability model. For inferential purposes, the traditional factor analysis coefficients $\tau$ and $\lambda$ are perhaps more convenient due to their standardized nature. 


\section{Structural Model}

If a theory is available proposing directional relations between (some of) the latent factors, then SEMs allow one to put a structure on the latent factors similar to what is done in path analysis with observed variables. These regressions among the latent factors are specfied in the structural part of the model:

$$
\begin{aligned}
& \eta=\Gamma \eta+\zeta \\
& \zeta \sim N(\mathbf{0}, \boldsymbol{\Xi}),
\end{aligned}
$$

where $\Gamma$ is a $D \times D$ matrix of regression coefficients where the diagonal elements are always zero, meaning that a factor cannot be explained by itself (hence $\boldsymbol{I}_{D}-\boldsymbol{\Gamma}$ is nonsingular). In the equation above, $\zeta$ is the $D \times 1$ residual vector of the regressions and is typically assumed to follow a multivariate normal distribution with mean zero and variance-covariance matrix $\boldsymbol{\Xi}$.

Hence to summarize, the measurement and structural models are similar to SEM for continuous observable variables, yet a threshold model is put in place to accommodate the categorical nature of the indicators.

\section{Copula Model for Coupled Measurement Residuals}

To allow for correlated measurement residuals in an SEM, we apply copulas to the latent continuous variables $\tilde{Y}_{p i}$ 's. Consider two of the underlying latent outcomes in the model, $\tilde{Y}_{p 1}$ and $\tilde{Y}_{p 2}$. Given their latent factor scores $\boldsymbol{\eta}_{p}$ and the assumption of logistically distributed measurement residuals $\varepsilon_{p 1}$ and $\varepsilon_{p 2}, \tilde{Y}_{p 1}$ and $\tilde{Y}_{p 2}$ each follow a univariate logistic distribution for the person $p, F_{\tilde{Y}_{p 1} \mid \boldsymbol{\eta}_{p}}\left(\tilde{y}_{p 1}\right)$ and $F_{\tilde{Y}_{p 2} \mid \boldsymbol{\eta}_{p}}\left(\tilde{y}_{p 2}\right)$.

In the traditional SEM as presented earlier, conditional independence is assumed, which gives rise to the multivariate joint distribution:

$$
\begin{aligned}
F_{\tilde{\boldsymbol{Y}}_{p} \mid \boldsymbol{\eta}_{p}}\left(\tilde{y}_{p 1}, \tilde{y}_{p 2}\right) & =\prod_{i=1}^{2} F_{\tilde{Y}_{p i} \mid \boldsymbol{\eta}_{p}}\left(\tilde{y}_{p i}\right) \\
& =C_{\Pi}\left(F_{\tilde{Y}_{p 1} \mid \boldsymbol{\eta}_{p}}\left(\tilde{y}_{p 1}\right), F_{\tilde{Y}_{p 2} \mid \boldsymbol{n}_{p}}\left(\tilde{y}_{p 2}\right)\right),
\end{aligned}
$$

where the product function is also known as the product copula $C_{\Pi}$ imposing independence between the margins. Accounting for correlated measurement residuals can then be done by applying to these univariate margins a copula function $C$ that allows for a suitable residual dependence structure:

$$
F_{\tilde{\boldsymbol{Y}}_{p} \mid \boldsymbol{n}_{p}}\left(\tilde{y}_{p 1}, \tilde{y}_{p 2}\right)=C\left(F_{\tilde{Y}_{p 1} \mid \boldsymbol{\eta}_{p}}\left(\tilde{y}_{p 1}\right), F_{\tilde{Y}_{p 2} \mid \boldsymbol{n}_{p}}\left(\tilde{y}_{p 2}\right) ; \delta\right),
$$

where $\delta$ refers to the parameter controlling the dependence. 


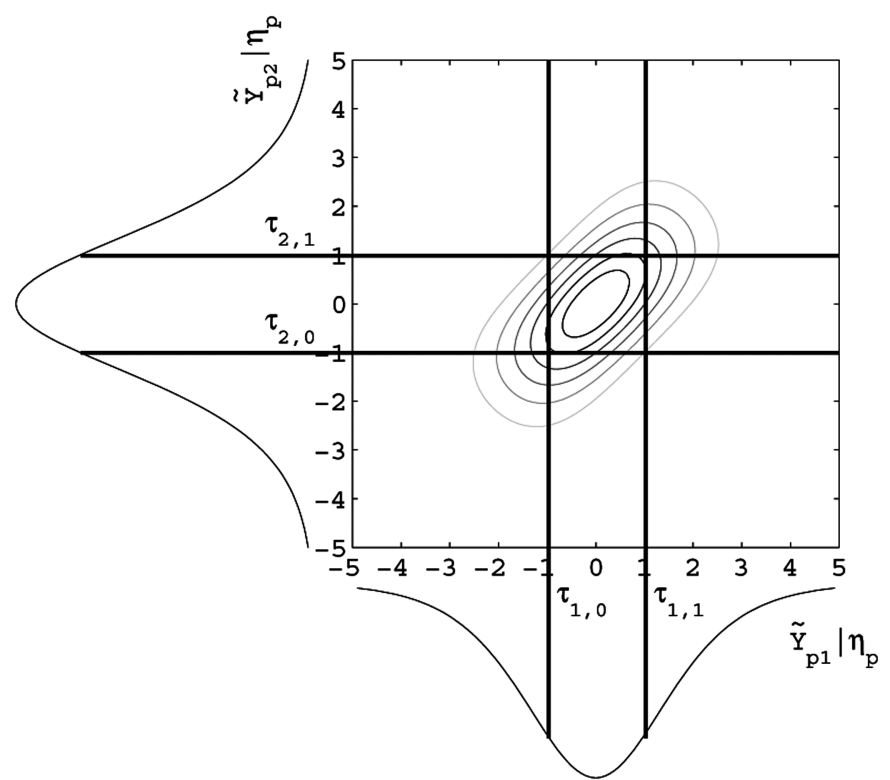

FIGURE 3 Copula constructed bivariate density with univariate logistic margins. Note. The solid vertical and horizontal lines refer to the category boundaries.

Figure 3 shows density contour lines from a resulting bivariate distribution with univariate logistic margins $F_{\tilde{Y}_{p 1} \mid \boldsymbol{\eta}_{p}}$ and $F_{\tilde{Y}_{p 2} \mid \boldsymbol{\eta}_{p}}$ and can be used to illustrate how one may calculate the joint probability distribution for the ordinal manifest outcomes $Y_{p 1}$ and $Y_{p 2}$. To find the joint probabilities, the volumes under the joint density delineated by the intersections of the category thresholds $\tau$ can be computed as follows:

$$
\begin{aligned}
& \operatorname{Pr}\left(Y_{p 1}=q_{1}, Y_{p 2}=q_{2} \mid \boldsymbol{\eta}_{p}\right)= \\
& \sum_{k_{1}=0}^{1} \sum_{k_{2}=0}^{1}(-1)^{k_{1}+k_{2}} C\left(F_{\tilde{Y}_{p 1} \mid \boldsymbol{\eta}_{p}}\left(\tau_{1, q_{1}+k_{1}}\right), F_{\tilde{Y}_{p 2} \mid \boldsymbol{\eta}_{p}}\left(\tau_{2, q_{2}+k_{2}}\right) ; \delta\right),
\end{aligned}
$$

where $F_{\tilde{Y}_{p i} \mid \boldsymbol{\eta}_{p}}\left(\tau_{i, q}\right)=1-G\left[-\frac{1}{\sqrt{\psi_{1}}}\left(\tau_{i, q+1}-\sum_{d=1}^{D} \lambda_{i d} \eta_{p d}\right)\right]$. Thus, the approach is based upon the computation of quadrant probabilities and can be generalized to any number of items.

In practice, the indicators $Y_{p i}(i=1, \ldots, I)$ will be divided in $S$ disjoint subsets $J_{s}$ of size $I_{s}$ according to their reference to a specific context or situation $s$ in the personality questionnaire. The joint probability of the outcomes in a 
context-specific subset $J_{s}$ will then be modeled by means of a copula-constructed joint distribution based upon the margins of the SEM and a specific copula function $C_{s}$, while conditional independence is assumed to hold between subsets, such that the joint probability model for the whole outcome vector $\boldsymbol{Y}_{p}$ becomes

$$
\operatorname{Pr}\left(\boldsymbol{Y}_{p}=\boldsymbol{y}_{p} \mid \boldsymbol{\eta}_{p}\right)=\prod_{s=1}^{S} \operatorname{Pr}\left(Y_{p i}=y_{p i}, i \in J_{s} \mid \boldsymbol{\eta}_{p}, C_{s}, \delta_{s}\right),
$$

where $\operatorname{Pr}\left(Y_{p i}=y_{p i}, i \in J_{s} \mid \boldsymbol{\eta}_{p}, C_{s}, \delta_{s}\right)$ is the joint probability derived from the joint cumulative distribution function $C_{s}\left(F_{\tilde{Y}_{p i} \mid \boldsymbol{\eta}_{p}}\left(\tilde{y}_{p i}\right), i \in J_{s} ; \delta\right)$ for the contextspecific indicator subset $J_{s}$.

\section{Dependence Structure}

In polytomous models, the issue of dependence is relatively complex. When considering the association between two variables $Y_{1}$ and $Y_{2}$ with $Q=3$ categories each, there are $(Q-1)^{2}=4$ global odds ratios to consider in the $3 \times 3$ cross-classification table:

\begin{tabular}{|c|c|c|}
\hline & 2 & 0 \\
\hline 2 & $O R 1$ & $O R 2$ \\
\hline 1 & $O R 3$ & OR4 \\
\hline
\end{tabular}

$$
\begin{aligned}
& O R_{1}=\frac{\operatorname{Pr}\left(Y_{1}=2, Y_{2}=2\right) \operatorname{Pr}\left(Y_{1}<2, Y_{2}<2\right)}{\operatorname{Pr}\left(Y_{1}=2, Y_{2}<2\right) \operatorname{Pr}\left(Y_{1}<2, Y_{2}=2\right)}, \\
& O R_{2}=\frac{\operatorname{Pr}\left(Y_{1}=2, Y_{2}>0\right) \operatorname{Pr}\left(Y_{1}<2, Y_{2}=0\right)}{\operatorname{Pr}\left(Y_{1}=2, Y_{2}=0\right) \operatorname{Pr}\left(Y_{1}<2, Y_{2}>0\right)}, \\
& O R_{3}=\frac{\operatorname{Pr}\left(Y_{1}>0, Y_{2}=2\right) \operatorname{Pr}\left(Y_{1}=0, Y_{2}<2\right)}{\operatorname{Pr}\left(Y_{1}<2, Y_{2}<2\right) \operatorname{Pr}\left(Y_{1}=0, Y_{2}=2\right)}, \\
& O R_{4}=\frac{\operatorname{Pr}\left(Y_{1}>0, Y_{2}>0\right) \operatorname{Pr}\left(Y_{1}=0, Y_{2}=0\right)}{\operatorname{Pr}\left(Y_{1}>0, Y_{2}=0\right) \operatorname{Pr}\left(Y_{1}=0, Y_{2}>0\right)} .
\end{aligned}
$$

Each global odds ratio is defined on one of the four binary cross-classifications of the full table. The set of odds ratios may show some regularities that are of substantive interest. Developing a model that has individual parameters to control each of these odds ratios would, however, be overcomplex and parsimonious structures need to be sought. Furthermore, in our setting, residual dependence might vary across categories but also across levels of the latent factors $\eta_{p d}$ as we are dealing with conditional dependence and hence conditional odds ratios. Depending on the specific copula, the predicted pattern of conditional associations may differ greatly.

On the level of the underlying continuous outcomes $\tilde{Y}_{p i} \mid \eta_{p}, i \in J_{s}$, the imposed dependence structure by each copula function is similar to the pattern as seen in Figure 1, only the margins would be logistic and hence have slightly heavier tails. Note that we do not use the Gaussian copula in this study as it requires the computationally intensive evaluation of the multivariate normal 
distribution in a categorical data context. Frank copula exhibits centralized and symmetric dependence, whereas the two other copulas show a form of tail dependence. Cook-Johnson copula exhibits strong lower tail dependence. Hence, this copula corresponds to a situation where the outcomes $\tilde{Y}_{p i} \mid \boldsymbol{\eta}_{p}, i \in J_{s}$ are known to be highly concordant at low values but somewhat less concordant at high values. In contrast, Gumbel-Hougaard copula exhibits strong upper tail dependence. Hence, Gumbel-Hougaard copula corresponds to a situation where the outcomes $\tilde{Y}_{p i}$ are known to be highly concordant at high values but somewhat less concordant at low values (for a more formal treatment of tail dependence, we direct the reader to Joe, 1997). It can be deduced that these tail dependence properties also transfer to the joint occurence of the discretized manifest outcomes. On the continuous latent level of $\tilde{\boldsymbol{Y}}_{p}$, the dependence parameter $\delta$ of each copula can be translated to a more familiar association measure as Kendall's $\tau$ rank correlation (see Appendix), which is formalized as the probability of concordance minus the probability of discordance (Nelsen, 1999). This derived measure can be used as a rough indicator of the general strength of association, but it should be kept in mind that it does not fully apply to the discrete case due to the presence of ties.

Because the copula works on the conditional SEM margins for ordinal data, the exact influence of the adopted copula function is differential according to $\boldsymbol{\eta}_{p}$, the person's latent factor scores. This implies that trait dependence and residual dependence are not necessarily independent as in the case of traditional multimethod multitrait models. For example, the residual association between a couple of indicators within a situation might be much stronger for generally aggressive persons than for nonaggressive persons. As such, different copula functions can accommodate different relationships with the latent traits. The tail dependence property again plays an important role here. A visual thought experiment in which one shifts the distributions in Figure 3 holding the thresholds fixed and looks at the changes in the covered surface for the different delineated rectangles (which reflect the probability of a specific joint outcome pattern) can shed light on these characteristics. To make things more concrete, consider again the two polytomous variables $Y_{1}$ and $Y_{2}$ that now follow marginal conditional probability functions with item discrimination $\alpha_{i d}$ fixed at 1 and difficulty parameters $\beta_{i 1}$ and $\beta_{i 2}$ set at -1 and 1 . In Table 1 we computed the set of conditional global odds ratios $\boldsymbol{O R}\left(\eta_{p}\right)$ for each of the three copulas for these two items with latent trait values varying from low $\left(\eta_{p}=-1.5\right)$ to high $\left(\eta_{p}=1.5\right)$ and copula dependence corresponding to a Kendall's $\tau$ of .35 . Note that when the local independence assumption holds all these odds ratios would in fact be equal to 1; the higher the odds ratio, the stronger the residual dependence. Whereas Frank copula $C_{F}$ shows a symmetrical dependence pattern that is relatively constant across the latent trait, the residual dependence between $Y_{1}$ and $Y_{2}$ increases with increasing values 
TABLE 1

Conditional Global Odds Ratios for Two Variables With $Q=3$ Categories Each

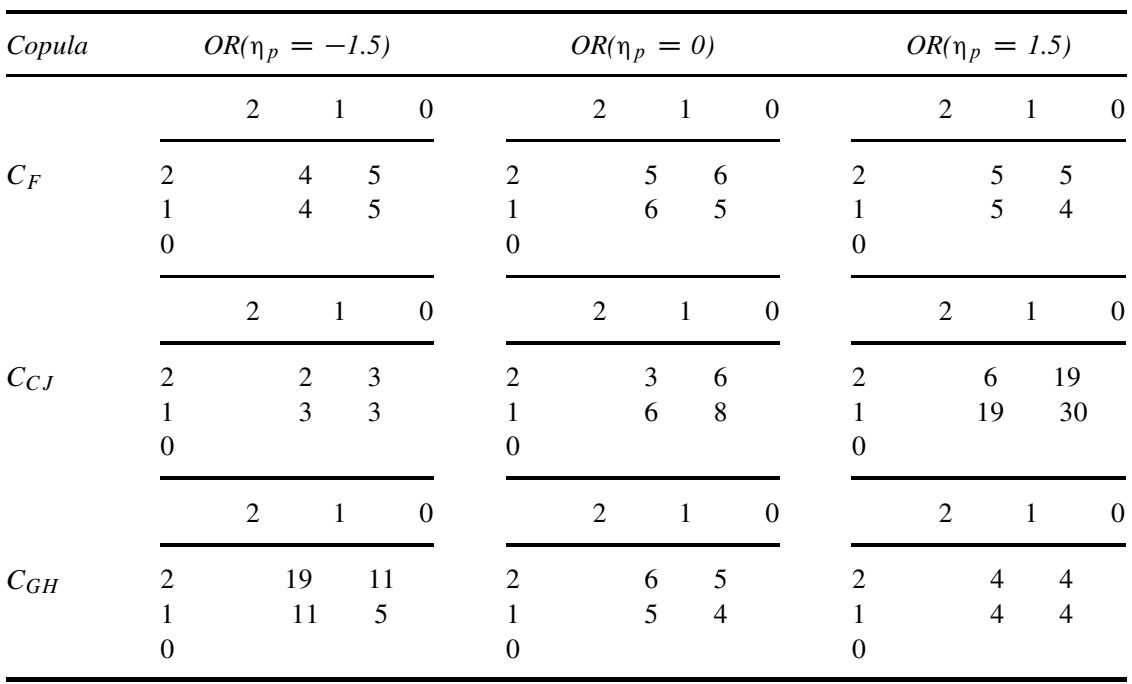

of the latent factor $\eta_{p d}$ for Cook-Johnson copula $C_{C J}$, whereas the opposite occurs for Gumbel-Hougaard copula $C_{G H}$ (residual dependence increases with decreasing latent factor scores). Notice that the tail dependence property transfers to the global odds ratios, with stronger association for the comparison $Y=0$ versus $Y>0$ than $Y<2$ versus $Y=2$ (see, e.g., pattern of odds ratios across the diagonal). Thus, the three copulas offer a variety of odds ratio structures yet without the cost of overparameterization as we chose copulas that are governed by a single dependence parameter homogeneous for the coupled indicator set.

\section{Estimation}

The estimation of the model requires a full information marginal maximum likelihood (MML) approach in which the latent factors $\boldsymbol{\eta}_{p}$ are integrated out. Because there are no closed form solutions for these integrals, they are approximated numerically using a Gauss-Hermite quadrature with 20 nodes for unidimensional models $(D=1)$ and 5 nodes per dimension for multidimensional models $(D>1)$. This MML method is suitably specified to the particularities of the categorical case, leading to consistent and efficient estimates, and the availability of a full likelihood expression allows for likelihood based inference (Bock, Gibbons, \& Muraki, 1988). Compared with traditional MML algorithms, only the computations for the joint outcome probabilities for copula subsets of indicators change. 
Although there are no well-established guidelines for which minimal conditions constitute an adequate result of the model fitting steps, a general approach is to establish that the model is identified, that the iterative estimation procedure converges, that all parameter estimates are within the range of permissible values, and that the standard errors of the parameter estimates have reasonable size (H. W. Marsh \& Grayson, 1995). We follow these recommendations.

\section{APPLICATION}

\section{Data and Research Questions}

Aggression is the basis of much societal and personal suffering (Berkowitz, 1993). As a consequence, the personality trait of aggression has been studied extensively in personality psychology. Over the years, researchers have come to conceptualize aggressiveness as consisting of several interrelated components that reflect the affective, behavioral, and cognitive aspects that are involved in aggression. This is the so-called ABC model (see, e.g., Martin, Watson, \& Wan, 2000). Recent definitional attempts and studies of aggressiveness consistently include three constructs: behavioral aggression, anger, and hostility. The behavioral aggression construct involves hurting or harming others and represents the instrumental component of aggressiveness. Anger includes physiological arousal and preparation for aggression and represents the affective component. Finally, hostility consists of feelings of ill will and injustice and represents the cognitive component of aggression. Yet, most questionnaires used in personality studies consider aggression at the general trait-level only, leaving out potential contextual effects in the assessments of aggression, contextual effects that evidently can be considered to exist (for an overview see, e.g., Anderson \& Bushman, 2002).

Our motivating data set arises from a study that assessed the three subcomponents of aggression in different situational contexts (Kuppens \& Tuerlinckx, 2007). As such, it allows us to study aggression while taking into account the context-dependence of aggressive behavior. In contrast, prior studies by Buss and Perry (1992) and Martin, Watson, and Wan (2000) were based upon typical general trait questionnaires. In particular, our example study assessed each aggression component in three different situational contexts: in the first situation, you find out that a friend spreads gossip and speaks ill about you behind your back. In the second situation, you are waiting for more than $30 \mathrm{~min}$ for the local waiter to bring the drink you ordered. In the third situation, you are out on a hiking trip with friends and the next day when setting up camp the construction poles of the tent appear to have been lost. This contextualized personality instrument was administered in a directed imagery study in which 
participants were asked to read each situational description, imagine as vividly as possible that they found themselves in this situation, and subsequently indicate to what extent they would display each of the three aggressive subcomponents.

The design of our aggression inventory is fully crossed ( 3 trait components $\times$ 3 situations), resulting in a total of nine indicators. The first set of three indicators refers to the first situation (gossip), the second set of three refers to the second situation (waiter), and the third set of three refers to the third situation (tent). Within such a set of three, the first question is an indicator of anger, the second an indicator of behavioral aggression, and the third an indicator of hostility. The inventory was completed by 340 university students who participated in the study in fulfillment of class requirements. To reduce the interpretational and computational problems associated with seven category items but at the same time retain basic ordinal information, we decided to collapse categories into three. Responses were coded into three ordinal categories from 0 to 2 , where increasing order indicates a higher judged plausibility of reacting in a manner like that suggested by the indicator.

With respect to these data, one crucial substantive question is how these components are interrelated. According to Buss and Perry (1992), anger is a psychological bridge between the cognitive component, hostility, and the instrumental component of aggression. Hostile thoughts and attributions are seen as crucial precursors to anger. In turn, anger often precedes aggressive behavior and makes the manifestation of such behavior more likely (Anderson \& Bushman, 2002). As such, the relation between hostility and behavioral aggression might be fully ascribed to the mediating role of anger, and we have a $\mathrm{CAB}$ process model. To test this process theory and the general $\mathrm{ABC}$ framework, four conceptual models are pitted against each other: a 1-dimensional aggression model, an orthogonal 3-dimensional $\mathrm{ABC}$ aggression model, an oblique 3dimensional $\mathrm{ABC}$ aggression model, and the mediation $\mathrm{CAB}$ process model. Figure 4 provides a visual summary of the four competing models using basic path diagrams (see, e.g., Bollen, 1989).

When ignoring or taking into account the additional dependencies coming from the contextualized nature of the measurement instrument, one might ask how this influences the inferences with respect to the research question. For instance, does accounting for the associations from the same situations change, in a qualitative or quantitative way, the directed relations between the $\mathrm{ABC}$ trait components? Furthermore, can we gain any additional information from the specific copulas that provide the best fit for the different contexts? We first report the results under the conventional conditional independence approach and ignore possible residual dependence issues. Next we contrast these reference results to the copula-added variants that explicitly model the dependence between measurement residuals that share the same contextual information. 


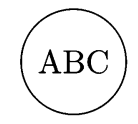

(A)

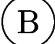

(a)

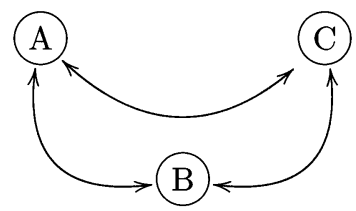

(c)

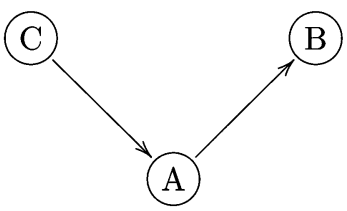

(d)

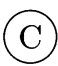

(b)

FIGURE 4 Path diagrams of the structural ABC models. (a) 1-dimensional. (b) Orthogonal ABC. (c) Oblique ABC. (d) CAB. A: Anger Affect, B: Behavioural Aggression, C: Hostility Cognition Single-headed arrows represent directional influences (i.e., regressions) between the latent traits and double-headed arrows represent correlations; the observed variables, residuals, and disturbances are omitted for clarity.

\section{Conditional Independence}

The likelihood ratio test between the nested oblique $\mathrm{ABC}$ model and the $\mathrm{CAB}$ process mediation model indicated that the two models did not differ significantly in model fit $\left(\chi^{2}=0.976, d f=1, p=.323\right)$, and thus we prefer the simpler mediation model. This indicates that our process theory can give a reasonable and more parsimonious account of the relations among the aggression components for the data at hand. In the left panel of Figure 5, the resulting standardized regression coefficients $\Gamma$ for the $\mathrm{CAB}$ model are given, and in Table 2, all parameter estimates for the chosen model are listed. We discuss these in more detail in the next subsection. To verify that our $\mathrm{ABC}$ formulation of aggression is not a needlessly complex model, the $\mathrm{CAB}$ mediation model was also compared with the 1-dimensional model in which no differentiation is made between the three $\mathrm{ABC}$ components of aggression. Model comparisons in terms of Aikaike's information criterion (AIC) among the four competing models favor the 1-dimensional model (see left panel of Table 3). Thus in principle, the ABC models should be rejected in favor of the more parsimonious 1-dimensional model, which would mean that the distinctions among anger, behavioral aggression, and cognitive hostility are not critical in understanding aggressiveness.

Note that these models all assumed that the latent factors $\eta_{p}$ are sufficient to explain the associations among the individual outcomes and hence that conditional independence holds; conditional on the latent factors, the individual 


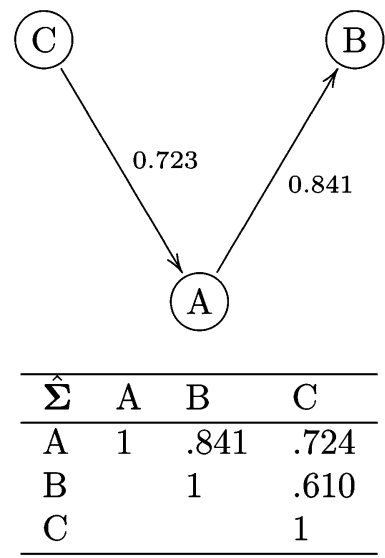

(a)
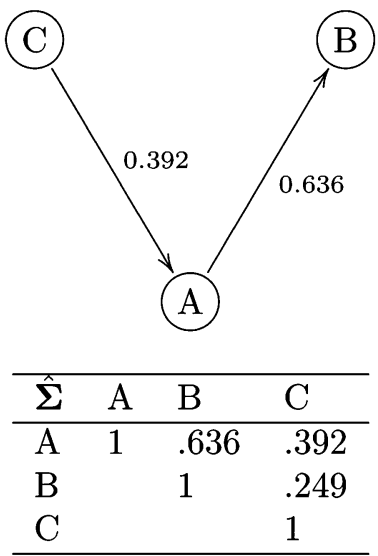

(b)

FIGURE 5 Path diagrams of the $\mathrm{CAB}$ vs the copula-CAB model. (a) CAB model ignoring context. (b) CAB model with copulas for context. A: Anger Affect, B: Behavioral Aggression, C: Hostility Cognition.

outcomes are independent. Conditional independence in the model should be reflected by uncorrelated measurement residuals $\varepsilon_{p i}$. However, if additional sources of association are present, this assumption does not hold, and there will be some residual dependence not explained by the model. As explained in the introduction, a possible approach to account for these residual dependencies is to add more latent factors. This means that, instead of having only the $\mathrm{ABC}$ personality traits, we can also include latent situation factors (i.e., a CT-CM model). However, a downside to this solution is that it may lead to overfactorization of a given data set and corresponding identification issues and increases the computational cost of fitting the model. In fact, we applied an $\mathrm{ABC}$ model with three added situation factors to the aggression inventory data, but the estimation algorithm failed to converge.

\section{Coupled Measurement Residuals}

Instead of adding latent variables to the model, an alternative approach is to allow for correlated measurement residuals. This is relatively straightforward when the residuals $\varepsilon$ are normally distributed because then one may allow for nonzero covariances in the covariance matrix of the residuals. Unfortunately, this approach only seems to work well for continuous normal outcomes and requires additional computational effort for categorical outcomes due to the extra multivariate (not closed form) integrals that are introduced in this way to 
TABLE 2

An ABC Model of Aggression: CAB Mediation Model

\begin{tabular}{lccccrcccccrr}
\hline$d$ & $s$ & $i$ & $\alpha_{i}$ & & \multicolumn{1}{c}{$\beta_{i l}$} & & $\beta_{i+}$ & & $\lambda_{i d}$ & $\tau_{i I}$ & \multicolumn{1}{c}{$\tau_{i 2}$} \\
\hline $\mathrm{A}$ & 1 & 1 & 1.234 & $(0.180)$ & -4.570 & $(0.166)$ & 2.510 & $(0.160)$ & 0.777 & -2.877 & -1.297 \\
$\mathrm{~B}$ & 1 & 2 & 1.124 & $(0.151)$ & -1.134 & $(0.112)$ & 2.232 & $(0.128)$ & 0.747 & -0.754 & 0.730 \\
$\mathrm{C}$ & 1 & 3 & 1.017 & $(0.184)$ & 0.253 & $(0.120)$ & 2.114 & $(0.170)$ & 0.713 & 0.178 & 1.661 \\
$\mathrm{~A}$ & 2 & 4 & 1.689 & $(0.180)$ & -1.318 & $(0.129)$ & 2.834 & $(0.157)$ & 0.861 & -0.671 & 0.772 \\
$\mathrm{~B}$ & 2 & 5 & 2.034 & $(0.210)$ & 1.217 & $(0.156)$ & 2.034 & $(0.204)$ & 0.897 & 0.537 & 1.434 \\
$\mathrm{C}$ & 2 & 6 & 1.401 & $(0.203)$ & 2.688 & $(0.198)$ & 1.810 & $(0.328)$ & 0.814 & 1.561 & 2.613 \\
$\mathrm{~A}$ & 3 & 7 & 1.348 & $(0.162)$ & -1.826 & $(0.118)$ & 2.430 & $(0.128)$ & 0.803 & -1.088 & 0.360 \\
$\mathrm{~B}$ & 3 & 8 & 1.496 & $(0.177)$ & -0.167 & $(0.124)$ & 2.161 & $(0.155)$ & 0.831 & -0.093 & 1.108 \\
$\mathrm{C}$ & 3 & 9 & 0.864 & $(0.188)$ & 1.991 & $(0.162)$ & 1.235 & $(0.209)$ & 0.654 & 1.506 & 2.440 \\
\hline
\end{tabular}

\begin{tabular}{ccccc} 
& & & $p$ & $\Gamma$ \\
\hline$g_{A C}$ & 1.074 & $(0.257)$ & 0.000 & 0.724 \\
$g_{B A}$ & 1.048 & $(0.240)$ & 0.000 & 0.841
\end{tabular}

Note. The parameter $\beta_{i+}=\beta_{i 2}-\beta_{i 1}$. The parameter $g_{Y X}$ is the unstandardized coefficient of the regression of $Y$ on $X$ ( $\Gamma$ is the standardized version). Standard errors are given between parentheses and $p$ values result from the Wald test. $d=$ aggression trait (A-affective, B-behavioral, $\mathrm{C}$ cognitive); $s=$ context (1-gossip, 2-waiter, 3 -tent); $i=$ indicator number.

the model. The same option for logistically distributed residuals, as considered in this article, is not readily available due to the absence of a general multivariate logistic distribution (see, e.g., Kotz, Balakrishnan, \& Johnson, 2000).

So far the specific contextualized structure of the aggression inventory was of no concern in our model analyses. The question now is, what happens to our

TABLE 3

Conditional Independence and Copula Versions of the Models of Interest

\begin{tabular}{lccccccc}
\hline & \multicolumn{3}{c}{ Conditional Independence } & & \multicolumn{3}{c}{ Copula } \\
\cline { 2 - 3 } Model & LogL & $\#$ Parameters & AIC & & LogL & $\#$ Parameters & AIC \\
\hline 1-dimensional & $-2,380.9$ & 27 & $4,815.8$ & $-2,350.0$ & 30 & $4,760.0$ \\
Orthogonal ABC & $-2,443.1$ & 27 & $4,940.2$ & $-2,350.3$ & 30 & $4,760.6$ \\
Oblique ABC & $-2,377.5$ & 30 & $4,815.0$ & $-2,322.7$ & 33 & $4,711.4$ \\
CAB mediation & $-2,377.9$ & 29 & $4,813.8$ & $-2,322.9$ & 32 & $4,709.8$ \\
\hline Null model & $-2,570.4$ & 18 & $5,176.7$ & & & & \\
Saturated model & $-1,819.8$ & 244 & $5,628.7$ & & & & \\
\hline
\end{tabular}

Note. The number of parameters in the saturated model corresponds to the number of unique observed response patterns in the sample data. 
model results and inferences when we do account for context by incorporating copula functions in the ordinal SEMs. Indicators belonging to the same context are coupled by means of a copula function, resulting in three subsets $J_{1}=$ $\{1,2,3\}, J_{2}=\{4,5,6\}$, and $J_{3}=\{7,8,9\}$, each with its own copula function $C_{s}$ with dependence parameter $\delta_{s}$.

Table 3 shows the model fit results of the four competing models as well as their corresponding copula extended versions. A first observation is that accounting for the contextualized structure leads to a strong increase in model fit, such that the copula variant is always retained in favor of the regular conditional independence SEM. Hence, the contextualized structure of the aggression inventory is indeed reflected in the dependence structure of the data. We cross fitted all possible combinations of copula types across the three subsets to verify which of the three copula functions under consideration provided the best fit for which situation. The best fitting set of copulas according to the model's log likelihood (i.e., Frank, Cook-Johnson, and Frank, for situations 1 to 3, respectively) remained constant across the models, indicating the stability of the additional dependence due to the situational contexts.

A second important observation is that accounting for the contextualized structure leads to a qualitative change in the chosen model. The difference between the 1-dimensional model and the $\mathrm{ABC}$ models is much clearer in the model with copula functions and now in favor of the latter $\left(\chi^{2}=54.1\right.$, $d f=2, p<.001)$ instead of the former. The related, but separable, factors of angry affect, behavioral aggression, and hostile cognition then reflect the fact that not all forms of aggression share similar manifestations and consequences. Among the $\mathrm{ABC}$ models, the model of choice according to a likelihood ratio test is the $\mathrm{CAB}$ mediation model $\left(\chi^{2}=.64, d f=1, p=.423\right)$. This implies that the results are in line with the mediation process theory as suggested by Buss and Perry (1992). In the right panel of Figure 5, the resulting standardized regression coefficients $\Gamma$ are indicated in a path diagram, and in Table 4, all parameter estimates for the preferred CAB model are listed.

Figure 5 demonstrates that the structural part of the conditional independence $\mathrm{CAB}$ model and the copula version of the $\mathrm{CAB}$ model show obvious quantitative differences. The effect of anger (A) on behavioral aggression (B) is less strong, as the value of the standardized regression coefficient decreases from $\Gamma_{B A}=$ 0.841 to $\Gamma_{B A}=0.635$. The effect of hostility (C) on anger (A) is also less strong, as shown in the value of the standardized regression coefficient that decreases from $\Gamma_{A C}=0.724$ to $\Gamma_{A C}=0.392$. Hence in our case, ignoring the contextual dependences leads to an overestimation bias of the structural relations. After the introduction of the copula functions, the ABC component's mutual interrelations are now corrected for with regard to the association accounted for by the contextual influences. 
TABLE 4

An ABC Model of Aggression: CAB Mediation Copula Model

\begin{tabular}{|c|c|c|c|c|c|c|c|c|c|c|}
\hline$d$ & $s$ & $\alpha_{i}$ & & $\beta_{i 1}$ & & $\beta_{i+}$ & & $\lambda_{i d}$ & $\tau_{i l}$ & $\tau_{i 2}$ \\
\hline A & 1 & 1.348 & $(0.179)$ & -4.690 & $(0.163)$ & 2.567 & 58) & 0.803 & -2.794 & -1.265 \\
\hline B & 2 & 1.235 & $(0.163)$ & -1.177 & (0.111) & 2.280 & 28) & 0.777 & -0.741 & 0.694 \\
\hline C & 3 & 2.834 & $(0.246)$ & 0.397 & (0.188) & 3.554 & 159) & 0.943 & 0.132 & 1.315 \\
\hline A & 4 & 1.717 & $(0.184)$ & -1.338 & $(0.123)$ & 2.852 & 54) & 0.864 & -0.673 & 0.762 \\
\hline B & 5 & 1.917 & $(0.201)$ & 1.156 & (0.143) & 1.978 & 99) & 0.887 & 0.535 & 1.450 \\
\hline $\mathrm{C}$ & 2 & 0.826 & $(0.191)$ & 2.264 & (0.173) & 1.645 & 08) & 0.637 & 1.746 & 3.014 \\
\hline A & 7 & 1.249 & (0.163) & -1.778 & $(0.107)$ & 2.367 & 19) & 0.781 & -1.111 & 0.368 \\
\hline B & 3 & 1.417 & $(0.176)$ & -0.156 & $(0.112)$ & 2.086 & 46) & 0.817 & -0.090 & 1.113 \\
\hline \multirow[t]{2}{*}{$\mathrm{C}$} & 9 & 0.800 & $(0.169)$ & 1.943 & $(0.150)$ & 1.207 & 06) & 0.625 & 1.517 & 2.459 \\
\hline & & & $p$ & $\Gamma$ & $J_{s}$ & Copula & $\delta$ & & $p$ & $\tau_{\text {Kendall }}$ \\
\hline$g_{A C}$ & 0.427 & $(0.116)$ & 0.000 & 0.392 & $1-1,2,5$ & $C_{F}$ & 2.882 & $(0.678)$ & 0.000 & 0.297 \\
\hline \multirow[t]{2}{*}{$g_{B A}$} & 0.757 & $(0.123)$ & 0.000 & 0.636 & $J_{2}=\{4,5,6$ & $C_{C J}$ & 1.506 & $(0.412)$ & 0.000 & 0.430 \\
\hline & & & & & $J_{3}=\{7,8,9$ & $C_{F}$ & 3.429 & $(0.553)$ & 0.000 & 0.344 \\
\hline
\end{tabular}

Note. The parameter $\beta_{i+}=\beta_{i 2}-\beta_{i 1}$. The parameter $g_{Y X}$ is the unstandardized coefficient of the regression of $Y$ on $X$ ( $\Gamma$ is the standardized version). Standard errors are given between parentheses and $p$ values result from the Wald test. $d=$ aggression trait (A-affective, B-behavioral, C—cognitive); $s=$ context (1-gossip, 2-waiter, 3-tent); $i=$ indicator number.

The threshold part of the conditional independence CAB model in Table 2 and the copula $\mathrm{CAB}$ model in Table 4 are rather similar. This observation could be anticipated because copula functions are a marginal modeling approach (see, e.g., Liang, Zeger, \& Qaqish, 1992) and impact the relational structure but not the mean structure. Looking at the pattern and values of the thresholds $\tau_{1}$ and $\tau_{2}$, it is noticeable that the most plausible reaction (i.e., with the lowest $\tau$ threshold values) is anger (A: average $\tau_{1}=-1.53$ and $\tau_{2}=-.05$ ), followed by behavioral aggression (B: average $\tau_{1}=-.10$ and $\tau_{2}=1.09$ ) and hostility (C: average $\tau_{1}=1.13$ and $\tau_{2}=2.26$ ), and this for all situations. Situation 1 (gossip: average $\tau_{1}=-1.13$ and $\tau_{2}=.25$ ) is the most salient context in which aggressive reactions are likely to be elicited, followed by situation 3 (tent: average $\tau_{1}=.11$ and $\tau_{2}=1.31$ ) and situation 2 (terrace: average $\tau_{1}=.54$ and $\tau_{2}=1.74$ ).

The factor loading part of the conditional independence $\mathrm{CAB}$ model in Table 2 and the copula $\mathrm{CAB}$ model in Table 4 showed more pronounced differences. Again this observation could be anticipated: because the factor loadings $\lambda_{i d}$ are a reflection of the model-implied underlying dependence structure, changes to this dependence structure by the addition of copulas to capture context effects will also show themselves here. The most noticeable differences occur for factor 3, the cognitive hostility trait (C). The factor loading of the indicator on hostility for the first situation is corrected upward from $\lambda_{i=3, d=3}=.713$ to $\lambda_{i=3, d=3}=.943$ 
and the factor loading of the indicator on hostility for the second situation is corrected downward from $\lambda_{i=6, d=3}=.814$ to $\lambda_{i=6, d=3}=.637$, reaching a level comparable to the factor loading of the third indicator of hostility. This illustrates that, when ignoring the context, we might wrongly conclude that the situation involving waiting on the terrace was the best indicator of hostility $\left(\lambda_{i=6, d=3}=.814\right)$, whereas after accounting for the contextual dependence the gossip situation is now considered the best indicator $\left(\lambda_{i=3, d=3}=.943\right)$ and the other two situations are relatively less indicative. The loadings on the other factors, anger and aggressive behavior (A and $\mathrm{B}$ ), remain relatively unchanged and all above .75 , which provides confidence in the quality of measurement of the latent traits.

When we look at the dependence parameter $\delta$ of all three copulas, they are significantly different from zero, indicating that the dependence structure deviates from the conditional independence structure. In our model for the contextualized aggression inventory, the degree of dependence imposed by a specific copula $C$, given its parameter $\delta$, can be seen as reflecting the consistency of response within a situation. It is an indication of additional dependence between responses given a person's relevant traits. A rough inspection of the Kendall's $\tau$ values points at situation 2 for being the situation where the most residual dependence is present and situation 1 for being the situation where the least residual dependence is present. Situation 2 is in fact the one with the most public control or constraint. Most people tend to react rather calmly when waiting a long time on a tavern's terrace, which can also be seen in the rather large estimated thresholds $\tau_{1}$ and $\tau_{2}$ for this situation's indicators $(i=4,5,6)$ compared with the other situations. This implies that people's reactions are largely determined by the context or environment and not only by their personal traits. In contrast, situation 1 involves a more up close and personal situation (people gossiping about you). In this type of situation, contextual influence is likely to be less pronounced. Frank copula fitted two out of three situations but not situation 2. The Cook-Johnson copula for situation 2 indicates that the residual dependence between the responses is larger here for more aggressive people. Given the public nature of situation 2 (waiting for your drink), it seems likely that this particular context will elicit either no reaction or, in contrast, once an aggressive person "snaps," a set of somewhat more aggressive responses. Hence, in this situation, the context response consistency is expected to be stronger for people who are in general already aggressive.

\section{DISCUSSION}

All in all, we concluded that the failure to account for the residual dependence due to the context gives rise to both qualitative and quantitative changes in 
related model inferences. If the contextualized nature of the aggression inventory is not explicitly modeled, no solid evidence was found to reject a unidimensional conceptualization of aggression in favor of the $\mathrm{ABC}$ conceptualization of aggression. Yet when the influence of contexts was acknowledged in the model, the results were clearly consistent with a mediation theory within the $\mathrm{ABC}$ framework. Furthermore, the copula approach also managed to provide extra information (see, e.g., response consistency) that allowed us to complete the overall picture of the personality study. In other words, context does matter in personality psychology, first as an important source of dependence and second because ignoring it can distort model inference.

Copula functions are a promising tool for multivariate model building in general and specifically in conjunction with SEM. The primary advantage is that copula functions keep the attractive framework of the existing structural equation methodology intact while at the same time offering new flexible dependence modeling options. The new flexibility allowed us to have method effects that vary across the latent trait dimension, surpassing the traditional orthogonality requirement. The extra freedom or flexibility that copula functions offer is positive, but also poses a challenge as it broadens the spectrum of modeling possibilities in the structural equation domain. The approach presented here is limited by the requirement of having disjoint subsets of indicators and a homogeneous within-set dependence structure. It might be of interest to investigate whether this approach can be extended to overlapping or nonhomogeneous subsets, although the restrictions needed to construct valid probability distributions might complicate matters in this area. With the introduction of the general multivariate modeling tool associated with the use of copulas, we hope to contribute to ongoing developments in the structural equation domain (see, e.g., in the context of simulation and robustness Mair, Satorra, \& Bentler, 2012).

\section{REFERENCES}

Anderson, C. A., \& Bushman, B. J. (20020. Human aggression. Annual Review of Psychology, 53, $27-51$.

Bartholomew, D. J., \& Knott, M. (1999). Latent variable models and factor analysis. London, UK: Hodder Arnold.

Berkowitz, I. (1993). Aggression: Its causes, consequences and control. New York, NY: McGrawHill.

Bock, R. D., Gibbons, R., \& Muraki, E. (1988). Full information factor analysis. Applied Psychological Measurement, 12, 261-280.

Bollen, K. (1989). Structural equations with latent variables. New York, NY: Wiley.

Braeken, J., \& Tuerlinckx, F. (2009). A mixed model framework for teratology studies. Biostatistics, $10,744-755$.

Braeken, J., Tuerlinckx, F., \& De Boeck, P. (2007). Copulas for residual dependency. Psychometrika, 72, 393-411. 
Buss, A. H., \& Perry, M. (1992). The aggression questionnaire. Journal of Personality and Social Psychology, 63, 452-459.

Clayton, D. G. (1978). A model for association in bivariate life tables and its application in epidemiological studies of familial tendency in chronic disease incidence. Biometrika, 65, 141151.

Cole, D., Ciesla, J., \& Steiger, J. (2007). The insidious effects of completely justifiable correlated residuals in latent variable covariance structure analysis. Psychological Methods, 12, 381-398.

Cook, R. D., \& Johnson, M. E. (1981). A family of distributions to modeling non-elliptically symmetric multivariate data. Journal of the Royal Statistical Society, Series B, 43, 210-218.

Eid, M. (2000). A multitrait-multimethod model with minimal assumptions. Psychometrika, 65, 241-261.

Eid, M., Nussbeck, F., Geiser, C., Cole, D., Gollwitzer, M., \& Lischetzke, T. (2008). Structural equation modeling of multitrait-multimethod data: Different models for different types of methods. Psychological Methods, 13, 230-253.

Frank, M. J. (1979). On the simultaneous associativity of $F(x, y)$ and $x+y-F(x, y)$. Aequationes Mathematica, 19, 194-226.

Gumbel, E. J. (1960). Distributions des valeurs extrêmes en plusieurs dimensions [Multidimensional extreme value distributions]. Publications de l'Institut de Statistique de l'Université de Paris, 9, 171-173.

Hougaard, P. (1986). A class of multivariate failure time distributions. Biometrika, 73, 671-678.

Joe, H. (1997). Multivariate models and dependence concepts. London, UK: Chapman \& Hall.

Kenny, D. (1976). An empirical application of confirmatory factor analysis to the multitrait-multimethod matrix. Journal of Experimental Social Psychology, 65, 507-516.

Kenny, D. A., \& Kashy, D. A. (1992). Analysis of the multitrait-multimethod matrix by confirmatory factor analysis. Psychological Bulletin, 112, 165-172.

Knol, D. L., \& Berger, M. P. (1991). Empirical comparison between factor analysis and multidimensional item response models. Multivariate Behavioral Research, 26, 457-477.

Kotz, S., Balakrishnan, N., \& Johnson, N. L. (2000). Continuous multivariate distributions (Vol. 1). New York, NY: Wiley.

Kuppens, P., \& Tuerlinckx, F. (2007). Personality traits predicting anger in self-, ambiguous-, and other-caused unpleasant situations. Personality and Individual Differences, 42, 1105-1115.

Lance, C., Noble, C., \& Scullen, S. (2002). A critique of the correlated trait-correlated method and correlated uniqueness models for multitrait-multimethod data. Psychological Methods, 7, $228-244$.

Liang, K.-Y., Zeger, S. L., \& Qaqish, B. (1992). Multivariate regression analyses for categorical data. Journal of the Royal Statistical Society, Series B, 54, 3-40.

MacCallum, R. (1986). Specification searches in covariance structure modeling. Psychological Bulletin, 100, 107-120.

Mair, P., Satorra, A., \& Bentler, P. (2012). Generating nonnormal multivariate data using copulas: Applications to SEM. Multivariate Behavioral Research, 47, 547-565.

Marsh, H. (1989). Confirmatory factor analysis of multitrait-multimethod data: Many problems and a few solutions. Applied Psychological Measurement, 13, 335-361.

Marsh, H. W., \& Grayson, D. (1995). Latent variable models of multitrait-multimethod data. In R. Hoyle (Ed.), Structural equation modeling: Concepts, issues and applications (pp. 1776-1198). Thousand Oaks, CA: Sage.

Martin, R., \& Watson, D., \& Wan, C. K. (2000). A three-factor model of trait anger: Dimensions of affect, behavior, and cognition. Journal of Personality, 68, 869-897.

Mischel, W. (1968). Personality and assessment. New York, NY: Wiley.

Mischel, W., \& Shoda, Y. (1995). A cognitive-affective system theory of personality: Reconceptualizing situations, dispositions, dynamics and invariance in personality. Psychological Review, 102, 246-268. 
Mood, A. M., Graybill, F. A., \& Boes, D. C. (1974). Introduction to the theory of statistics. New York, NY: McGraw-Hill.

Nelsen, R. B. (1999). An introduction to copulas. New York, NY: Springer.

Rindskopf, D. (1984). Structural equation models: Empirical identification, Heywood cases, and related problems. Sociological Methods Research, 13, 109-119.

Salhi, S. (1998). Heuristic search methods. In G. A. Marcoulides (Ed.), Modern methods for business research (pp. 147-175). Mahwah, NJ: Erlbaum.

Sklar, A. (1959). Fonctions de répartition à $n$ dimension et leurs marges [Distribution functions in $n$ dimensions and their margins]. Publications Statistiques Université de Paris, 8, 229-231.

Skrondal, A., \& Rabe-Hesketh, S. (2004). Generalized latent variable modeling: Multilevel, longitudinal and structural equation models. Boca Raton, FL: Chapman \& Hall/CRC.

Steiger, J. H. (1990). Structural model evaluation and modification: An interval estimation approach. Multivariate Behavioral Research, 25, 173-180.

Takane, Y., \& de Leeuw, J. (1987). On the relationship between item response theory and factor analysis of discretized variables. Psychometrika, 52, 393-408.

Vansteelandt, K., \& Van Mechelen, I. (1998). Individual differences in situation behavior profiles: A triple typology model. Journal of Personality and Social Psychology, 75, 751-765.

Wirth, R. J., \& Edwards, M. C. (2007). Item factor analysis: Current approaches and future directions. Psychological Methods, 12, 58-79.

Wothke, W. (1996). Models for multitrait-multimethod matrix analysis. In G. A. Marcoulides \& R. E. Schumacker (Eds.), Advanced structural equation modeling: Issues and techniques (pp. 7-56). Mahwah, NJ: Lawrence Erlbaum Associates.

\section{APPENDIX: COPULA FUNCTIONS}

We limit ourselves here to a basic set of copula functions that span a diverse set of dependence structures and restrict their formulation to homogeneous dependence structures. Note that more involved dependence structures are possible, but these are functionally more complex and computationally less straightforward.

1. The Product copula, which corresponds to the basic independence case

$$
C_{\Pi}\left(U_{1}, \ldots, U_{I}\right)=\prod_{i=1}^{I} U_{i} .
$$

2. The Gaussian copula, which is based upon the multivariate normal distribution

$$
C_{\Phi}\left(U_{1}, \ldots, U_{I} ; \delta\right)=\Phi_{I}\left(\Phi^{-1}\left(U_{1}\right), \ldots, \Phi^{-1}\left(U_{I}\right) ; \delta\right),
$$

where $\Phi^{-1}$ is the inverse normal univariate $\mathrm{CDF}$ and $\Phi_{I}$ the $I$-variate normal distribution. The dependence parameter $\delta$ is also known as the Vanderwaerden signed rank correlation and falls in the interval $[-1,1]$ with the classical correlation interpretation that remains intact. Note that Kendall's $\tau=\operatorname{asin}(\delta) * 2 / \pi$. 
3. The Frank copula (Frank, 1979)

$$
C_{F}\left(U_{1}, \ldots, U_{I} ; \delta\right)=\frac{-1}{\delta} \log \left(1-\frac{\prod_{i=1}^{I}\left(1-\exp \left(-\delta U_{i}\right)\right)}{\prod_{i=1}^{I-1}(1-\exp (-\delta))}\right),
$$

where $\delta$ is restricted to the interval $] 0, \infty[$. As $\delta$ aproaches 0 , the margins become independent (and hence $C \rightarrow C_{\Pi}$ ). As $\delta$ approaches $\infty$, the copula approximates the upper bound of positive association between the set of random variables. Note that Kendall's $\tau=1+4\left(D_{1}(\delta)-1\right) / \delta$, where $D_{1}$ is the first Debye function, $D_{1}(x)=\frac{1}{x} \int_{0}^{x} \frac{t}{\exp (t)-1} d t$.

4. The Cook-Johnson copula (Clayton, 1978; Cook \& Johnson, 1981)

$$
C_{C J}\left(U_{1}, \ldots, U_{I} ; \delta\right)=\left(\sum_{i=1}^{I} U_{i}^{-\delta}-I+1\right)^{\frac{-1}{\delta}}
$$

with $\delta$ restricted to the interval $] 0, \infty[$. As $\delta$ aproaches 0 , the margins become independent $\left(C_{\Pi}\right)$. As $\delta$ approaches $\infty$, the copula approximates the upper bound of positive association. Note that Kendall's $\tau=\delta /(2+\delta)$.

5. The Gumbel-Hougaard copula (Gumbel, 1960; Hougaard, 1986)

$$
C_{G H}\left(U_{1}, \ldots, U_{I} ; \delta\right)=\exp \left(-\left[\sum_{i=1}^{I}\left(-\log \left(U_{i}\right)\right)^{\delta}\right]^{\frac{1}{\delta}}\right)
$$

with $\delta$ restricted to the interval $] 1, \infty[$. The boundary values of this interval, 1 and $\infty$, correspond to $C_{\Pi}$ and the maximum positive association, respectively. Note that Kendall's $\tau=1-1 / \delta$. 